\title{
MAIN PULSE-INTERPULSE CORRELATION AND MODE CHANGING IN PSR 1055-52
}

\author{
JAMES D. BIGgS \\ Nuffield Radio Astronomy Laboratories, University of Manchester
}

\begin{abstract}
We have studied the individual pulses from PSR 1055-52, a pulsar which has a strong interpulse. Their properties, and those of the average pulse profile are consistent with an emission geometry in which the magnetic and rotation axes are nearly perpendicular. That is, we are observing both magnetic poles of this pulsar. A correlation between the emission from the two magnetic poles was found wherein a strong interpulse is followed by a strong main pulse. Also, the main pulse appears to undergo mode changes that are primarily characterized by a change in the peak flux density of component I.
\end{abstract}

\section{Introduction}

Radio pulsar PSR 1055-52 has a relatively short period $(197 \mathrm{~ms})$, and its period derivative $\left(5.8 \times 10^{-15} \mathrm{~s} \mathrm{~s}^{-1}\right)$ is near the mean for the pulsar population. These parameters imply that it is quite young with a characteristic age of $5.4 \times 10^{5} \mathrm{yr}$. A multifrequency study of its average pulse profile by McCulloch et al. (1976) found a strong interpulse approximately midway between main pulses. They also found that its pulse profile, when scaled appropriately in pulse period, was similar to that of PSR 0531+21, the Crab Nebula pulsar.

PSR 1055-52 is somewhat unusual in that the energy in its interpulse is very similar to that in the main pulse over a wide range of radio wavelengths McCulloch et al. (1976). This property facilitates the study of any related emission variations between these pulse components. Also, the main pulse-interpulse separation is constant from $60 \mathrm{MHz}$ to $1.4 \mathrm{GHz}$ (Turner 1980) and indicates that the observed emission is emanating from both magnetic poles of the pulsar. The polarization position angle study of Lyne and Manchester (1988) confirms this, and they estimate that the angle between the rotation and magnetic axes is $\approx 75^{\circ}$. Thus the study of any main pulse-interpulse interaction gives valuable insight into the emission mechanisms above each pole, and their 'global' interaction.

\section{Observations}

The observations of PSR 1055-52 described in this paper were obtained using the 64-m Parkes telescope operating at $645 \mathrm{MHz}$ with a right-hand circularly polarized feed. The hardware is described more fully by Biggs et al. (1985). Ten data sets were recorded at 400 samples per pulse period $(\approx 0.5 \mathrm{~ms})$ and had a post-detection time constant of $0.5 \mathrm{~ms}$.

\section{Average profile}

The average pulse profile derived from all the 645MHz data from PSR 1055--52 is plotted in figure 1. This was calculated by normalizing the average profile from each data set, then aligning component II with $90^{\circ}$ longitude and then averaging all these with a weighting equal to the number of pulses recorded in the data set. Three components are clearly visible in the main pulse (components I to III), and the interpulse has at least three components. There is no evidence for a bridge of emission between the main pulse and interpulse since the difference between the two off-pulse regions is $<0.01$ times the peak flux density (Biggs et al. 1988) (also see figure 1).

A comparison of the published average profiles for PSR 1055-52 reveals that they are unstable over relatively long time scales. The relative strengths of the main pulse components and their separations are given in table 1 . This clearly indicates that the relative strengths of the components in the main pulse change, while their positions remain unchanged within the given uncertainties. A detailed comparison between all the interpulse average profiles is not possible because of the low $S / N$ of the earlier work, however the interpulse presented by McCulloch et al. (1978) has a similar morphology to that in figure 1. The separation between the centroids of the main pulse and interpulse remained constant at $\approx 155^{\circ}$.

Variations in the relative strengths of the pulse components were also seen in the profiles obtained from the $645-\mathrm{MHz}$ data sets. Changes in the relative amplitude of the components of the main pulse were clearly apparent and, to a lesser degree, in the interpulse. Generally, component II has the largest peak fux density and component I the greatest variability. Changes in the component separations do 


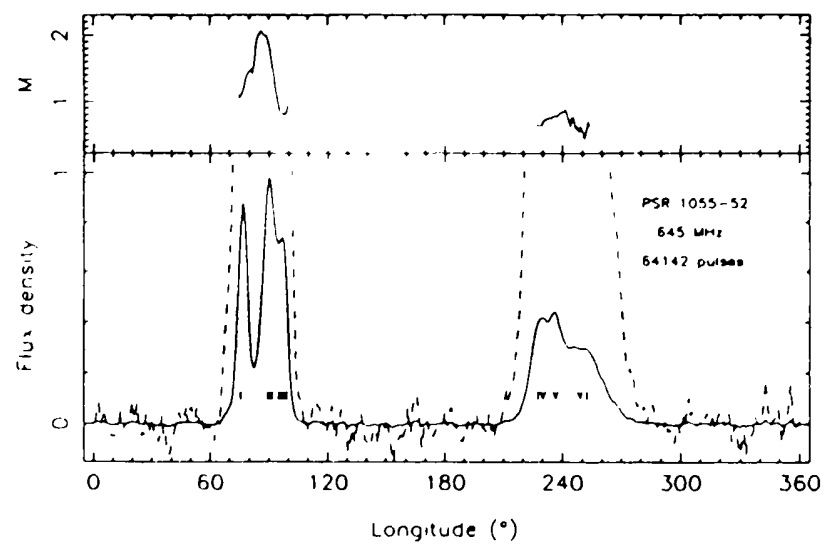

Figure 1 Lower panel: the average pulse profile for PSR 1055-52 at $645 \mathrm{MHz}$ (solid line). The components are labeled at the approximate position of their centroids. The sample interval and time constant smoothing are $0.9^{\circ}(0.5 \mathrm{~ms})$ and the dispersion broadening is much less than this. Also plotted (dashed line) is the average pulse profile multiplied by a factor of 10 in order to detect any possible bridges of emission between the main pulse and interpulse.

Upper panel: Modulation index plotted as a function of longitude for the sequence of 2460 pulses that had the highest $\mathrm{S} / \mathrm{N}$ of all the data presented here.

Table 1 Observations of the main pulse of PSR 1055-52 near $640 \mathrm{MHz}$

\begin{tabular}{|lr|rrr|}
\hline date & & \multicolumn{3}{|c|}{ May '72- } \\
& Nov'72 & May '76- & May '81 \\
\hline no. of pulses & & $?$ & 12275 & 64142 \\
& & & & \\
rank & I & 3 & 1 & 2 \\
of & II & $2^{\dagger}$ & $3^{\dagger}$ & 1 \\
component & III & $1^{\dagger}$ & $2^{\dagger}$ & 3 \\
strength & & & & \\
& & & & \\
separation & I-II & $15 \pm 1$ & $13.5 \pm 1$ & $13.5 \pm 0.9$ \\
& II-III & $6 \pm 1$ & $6.6 \pm 1$ & $6.3 \pm 0.9$ \\
reference & & & & \\
\hline
\end{tabular}

'Ranks are the same given the uncertainties in the data. References: 1 McCulioch et al. 1976, 2 McCulloch et al. 1978,3 this paper.

not appear to accompany these amplitude variations, but the longitude resolution of the observations $\left(0.9^{\circ}\right)$ is rather poor compared to the widths of the components $\left(\approx 5^{\circ}\right)$. The changes in relative amplitude were further studied by forming average profiles from $2000 P$ (pulse period) subsets of the data sets. The information obtainable from these is limited because of their poor S/N. Generally the profiles were stable; however, some changes in relative amplitude were detected from one $2000 P$ integration to the next, indicating that the time scale for change is $\lesssim 2000 P$. No correlations were detected between the shape of the main pulse and that of the interpulse.

\section{Individual pulse properties}

The analysis of individual pulses was rather limited owing to their low S/N. Only about 2500 pulses had sufficient $S / N$, and from these it appears that subpulses of the main pulse are more sporadic than those from the interpulse. This is borne out by the modulation index $\left[=\left(\sigma_{\text {on }}^{2}-\sigma_{\text {off }}^{2}\right)^{\frac{1}{2}} /\left(\left\langle I_{\text {on }}\right\rangle-\left\langle I_{\text {off }}\right\rangle\right)\right.$, where $\sigma^{2}$ is the variance of the flux density, $\langle I\rangle$ is the average flux density, and, "on" and "off" refer to the on-pulse or off-pulse part of the average profile] shown in figure 1. The fluctuation spectra of the energy in most of the components were generally flat or had a slight monotonic decrease with increasing fluctuation frequency. However, component $\mathrm{V}$ has a small peak $(\approx 3 \mathrm{~dB}$ above the average por.$c)$ at frequency 0.045 cycles $/ P$ with $Q(=\nu / \Delta \nu) \approx 1.5$, and component II has a broad ft ture $(\approx 3 \mathrm{~dB}$ above the average power) at $0.1 \mathrm{cy}$ ics $/ P$ with $Q \approx 0.6$. There were no apparent correlations between the fluctuation spectra for each data set and the corresponding average-pulse profile shape.

The correlation between the energy from the main pulse and interpulse was examined on a time scale of $1 P$ in each of the data sets using a timelagged Spearman's rank cross-correlation coefficient (see Daniel 1978). This correlation coefficient has the virtue of being relatively unaffected if the data set does not have a normal distribution. Before correlation, long-term fluctuations were removed by subtracting the running mean of the off-pulse region for the current and previous 24 pulse periods from each pulse. Figure 2 shows the correlation between the energy of the main pulse and interpulse for the 10 data sets from PSR 1055-52. The peak correlation coefficients are about 0.15 and have a significance $>0.999$. More importantly, they occur most frequently at $-1 P$ lag indicating that a strong interpulse is followed by a strong main pulse.

Correlations were also sought between the energy of the components of PSR 1055-52. They were not as significant as those in figure 2 because of the lower $\mathrm{S} / \mathrm{N}$ of the data, but generally gave the expected correlations. That is, the main pulse components had a maximum correlation with the interpulse components at $-1 P$ lag, while correlations within the main pulse or interpulse were maximum at $0 P$ lag. The most significant correlations occurred for component II of the main pulse and component $\mathrm{V}$ of the interpulse.

\section{Discussion}

A two-pole model seems to best explain many of the radio-frequency properties of PSR 1055-52. However, correlated activity between the main pulse and 


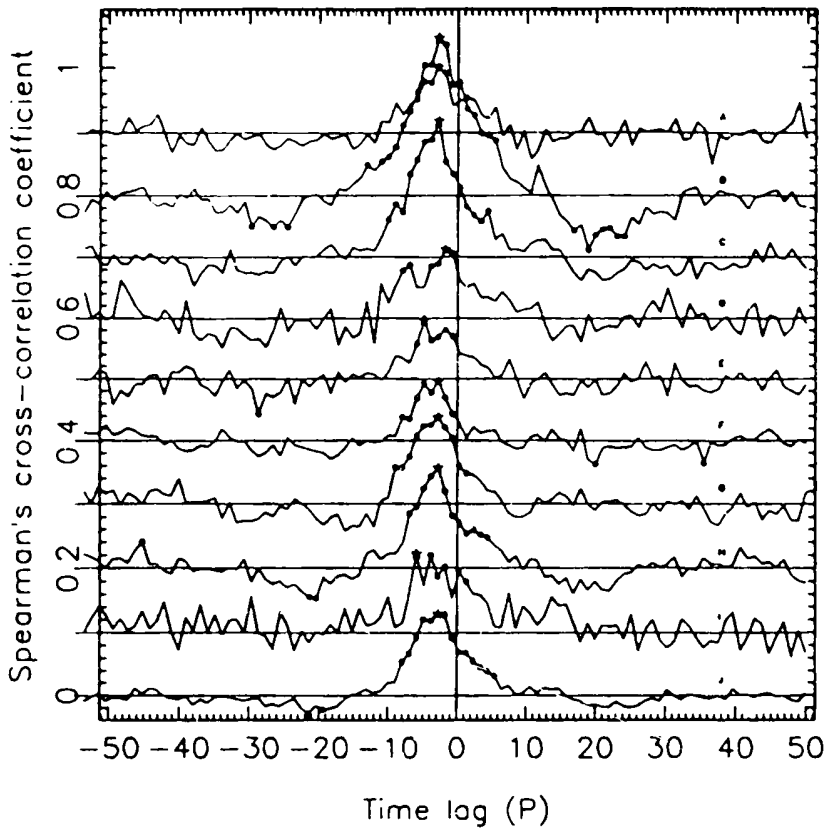

Figure 2 Time-lagged Spearman's cross-correlation coefficient between the energy in the main pulse and interpulse of PSR 1055-52 for 10 data sets. The cross-correlation peaks $(\star)$ and all the points that have a statistical significance $>0.999(\bullet)$ are plotted. Zero lag corresponds to aligning the main pulse with the interpulse, and the positive sense of lag brings the main pulse into alignment with later interpulses. The cross-correlations are separated by 0.1 for clarity.

interpulse is easier to understand if these pulse components are associated with only one pulsar magnetic pole. The supporting evidence for a singlepole geometry in PSR 1055-52 is based on only one observed property, namely, that the main pulseinterpulse separation is significantly less than the $180^{\circ}$ separation between the poles. There is substantially more evidence for a two pole geometry:

1. The main pulse-interpulse separation is constant from $60 \mathrm{MHz}$ to $1.4 \mathrm{GHz}$ (Turner 1980).

2. When the evolution of beamwidth with pulse period is taken into account (Lyne and Manchester 1988, Biggs 1990a) the main pulse and interpulse have widths that are not dissimilar to those observed in the vast majority of pulsars-namely, the widths at half power are $26^{\circ}$ and $35^{\circ}$ (McCulloch et al. 1978), respectively, and the components within them have widths $5^{\circ}$ to $10^{\circ}$.

3. Any bridge of emission connecting the main pulse and interpulse has a peak flux density $<1$ per cent of the peak flux density of the main pulse (Biggs et al. 1988),

4. Extremely good fits to the Radhakrishnan and Cooke (1969) model of the polarization position angle were obtained by Lyne and
Manchester (1988) with an angle of $\approx 75^{\circ}$ between the magnetic and rotation axes.

5. From a study of the core-component emission geometry Rankin (1990) has calculated that the angle between the magnetic and rotation axes is $\approx 90^{\circ}$.

The $155^{\circ}$ main pulse-interpulse separation may be accommodated by the two-pole model if we assume that the trailing edge of the interpulse is missing, probably as a result of the patchy nature of the emission (Lyne and Manchester 1988). Two observed properties of PSR 1055-52 confirm this interpretation. First, component VI appears to be positioned at the center of symmetry of the emission beam associated with the interpulse since it is coincident with the point of inflection of the polarization position angle (Lyne and Manchester 1988). Also, the fluctuation spectrum of component $V$ is consistent with that exhibited by conal (from a concentric ring aligned with the magnetic axis, see Rankin 1983) emission (Rankin 1986). Thus component $V$ is positioned near the edge of the pulsar emission beam and not at its center as suggested by its position in the observed interpulse.

The two-pole emission geometry implies that the correlation between activity in the main pulse and interpulse for PSR 1055-52 results from some form of communication between the two magnetic poles of this neutron star. The maximum crosscorrelation occurs at $-1 P$ and implies that the time of flight along the communication channel is less than or equal to the temporal separation of an interpulse with the next main pulse, that is, $\approx 112 \mathrm{~ms}$. The communication channel might be the open magnetic field lines or the neutron-star surface. Both these possibilities will be discussed below. In the extremely unlikely circumstance that the inferred geometry is incorrect-that is, we are observing only one magnetic pole, the correlation might be the result of the circulation of a 'spark' (that triggers radio emission) around a magnetic pole. The time scale of such a circulation does not, however, fit the observations. Ruderman and Sutherland (1975) predict that the circulation period of a spark is $P_{3} \approx 6 B_{12} P^{-1}$, where $B_{12}$ is the surface magnetic field in units of $10^{12} \mathrm{G}$, and $P$ is the pulsar period. For the parameters of PSR 1055-52 this predicts $P_{3} \approx 34 P$, which is more than an order of magnitude larger than the observed $-1 P$ correlation.

If the communication is via the open field lines then the time taken for particles to move from one polar cap to the other along the field lines depends on the component position (or particular bundle of field lines) within the emission cone (Biggs 1990a). Components I, III and IV are probably close to 
the trailing or leading edges of the emission cone, and for them the minimum communication time $\tau_{\mathrm{p}-\mathrm{p}} \approx 0.44 \mathrm{P}$, or $87 \mathrm{~ms}$. Component II, however, has the strongest correlation with components in the interpulse and, because the source of this component is located on field lines which extend well beyond the light cylinder, the minimum communication time is much larger than that observed. However, as noted by Biggs (1990b), it is probably unlikely that a signal can pass through the light cylinder since its azimuthal velocity will, by definition, equal $c$.

An alternative medium for communication between the two magnetic poles is the surface of the neutron star. The polar-cap surface is probably intimately related to the emission mechanism since it is continually bombarded by either very high energy electrons or positrons produced by pair-production in the strong pulsar magnetic field (Jones 1978, Cheng and Ruderman 1980). The high magnetic field at the neutron-star surface drastically reduces the thermal conductivity perpendicular to the field (Ruderman 1971) and thus rules out a communication channel based on thermal transport through the neutron-star crust. However, neutron stars can support a wide variety of nonradial oscillations which may facilitate the observed communication.

Van Horn and co-workers (McDermott et al. 1988 and the references therein) have analysed the nonradial oscillations of neutron stars and determined the periods and damping times of the many possible modes. They have calculated that the $g$ modes in the crust have periods on the order of $100 \mathrm{~ms}$, and have electromagnetic damping times smaller than or nearly equal to these periods in a $10^{12} \mathrm{G}$ magnetic field. Communication between the magnetic poles may proceed as follows. Bombardment at one pole (associated with the radio emission process) may induce morphological changes of the polar cap (Vivekanand and Radhakrishnan 1980). These changes may excite crustal g-modes because they have low pulsation energies (McDermott, Van Horn, and Hansen 1988). The oscillations travel through the crust with a speed somewhat greater than the speed of shear waves and so should arrive at the other polar cap after $\lesssim 2 \mathrm{~ms}$ (McDermott, Van Horn, and Hansen 1988). This may induce morphological changes in the other polar cap which may couple to the radio emission mechanism, and possibly produce a detectable change in the emission. The short damping time of the g-modes means that, while there is enough time for communication, the excitation is short lived, and thus the interaction does not continue for many cycles which might possibly have the effect of masking the communication.

The activity in the interpulse and then the main pulse of PSR 1055-52 is a one-way communication. This implies that an asymmetry exists between the two magnetic poles and also argues against an external agency. The most obvious asymmetry is the polarity of the magnetic poles. However, present pulsar models are not sufficiently developed to indicate how this asymmetry couples to the polar cap.

The average pulse profiles for some pulsars with complex average pulse shapes tend to stabilize only after $\approx 2000$ pulse periods (Manchester and Taylor 1977). This relatively slow stabilization is due to mode changing, wherein the average profile changes to another stable form for up to about 1000 pulses and then reverts to its normal form. The change in the average pulse profile for the main pulse of PSR 1055-52 is most probably the result of a mode change that is primarily characterized by an approximately 50 percent increase in the flux density of component I for $\approx 2000$ pulse periods. The mode changing in PSR 1055-52 is somewhat unusual because it is not accompanied by other changes that have been found by Bartel et al. (1982) to be strongly associated with this phenomenon, namely,

1. the appearance of new components,

2. changes in the longitude of components, and

3. changes of the fluctuation properties.

Also, while the ratio of the energy from the main pulse to that from the interpulse remained fairly constant, there were significant variations. This probably reflects a change in the relative energy output from the two magnetic poles and cannot be described as a mode change since this phenomenon refers to changes within the whole of the emission region and/or polar cap of one magnetic pole.

For a more detailed discussion of the matters raised here the reader is referred to the recent work by Biggs (1990b). 